\title{
Curriculum scientifique et innovation
}

Elisa Bonilla, Armando Sánchez, Teresa Rojano et José Antonio Chamizo Traducteur : Noël Groult

\section{OpenEdition}

\section{Journals}

Édition électronique

URL : http://journals.openedition.org/ries/3341

DOI : 10.4000/ries.3341

ISSN : 2261-4265

Éditeur

Centre international d'études pédagogiques

Édition imprimée

Date de publication : 1 juin 1997

Pagination : 10-15

ISSN : 1254-4590

Référence électronique

Elisa Bonilla, Armando Sánchez, Teresa Rojano et José Antonio Chamizo, «Curriculum scientifique et innovation », Revue internationale d'éducation de Sèvres [En ligne], 14 | 1997, mis en ligne le 30 juillet 2013, consulté le 19 avril 2019. URL : http://journals.openedition.org/ries/3341 ; DOI : 10.4000/ ries.3341

Ce document a été généré automatiquement le 19 avril 2019

(c) Tous droits réservés 


\title{
Curriculum scientifique et innovation
}

\author{
Elisa Bonilla, Armando Sánchez, Teresa Rojano et José Antonio Chamizo \\ Traduction : Noël Groult
}

1 La réforme des programmes au Mexique n'est pas un cas isolé ; plusieurs pays se sentent insatisfaits des résultats de l'enseignement des sciences, des mathématiques et de la technologie dans leur système scolaire. C'est pour cette raison qu'ils favorisent des projets innovants dont le but est de transformer l'enseignement de ces matières. Selon une étude sur vingt-trois projets, réalisés dans treize pays développés membres de l'OCDE, l'idée qu'une amélioration de l'enseignement dans ces trois domaines apportera des bénéfices sur le plan individuel pour l'épanouissement de l'élève et pour la communauté à laquelle il appartient est considérée comme une raison fondamentale pour le développement de ces innovations. Ces bénéfices sont souvent interprétés en termes d'une plus grande capacité de concurrence économique dans un monde " globalisé »3.

2 S'il est vrai que les changements proposés et les projets qui les encadrent sont très divers quant à la forme et à la portée, l'étude a trouvé beaucoup de ressemblances entre eux, tant en ce qui concerne les thèmes choisis que les méthodes d'enseignement. Black, Atkin et Atkin établissent trois aspects communs à pratiquement tous les projets curriculaires de rénovation qu'ils ont étudiés :

- l'importance du travail pratique réalisé par les élèves, dans une proportion bien supérieure à celle qu'il avait dans le passé ;

- l'explicitation des relations aussi bien entre les sciences elles-mêmes qu'entre les sciences et d'autres domaines de la connaissance (objectif principal du processus d'enseignement) ;

- la reconnaissance, à travers les approches de l'enseignement, du fait que les sciences et les mathématiques sont des manières de connaître et d'expliquer le fonctionnement du monde qui nous entoure.

3 La réforme mexicaine des programmes de sciences naturelles et de mathématiques partage ces trois ambitions. 


\section{Mathématiques}

4 Le courant structuraliste, en vogue dans les années soixante dans le domaine des mathématiques, a eu une grande influence sur les réformes d'éducation de la fin de cette décennie au niveau mondial. Une des manifestations de cette influence est, par exemple, la conception de l'algèbre symbolique dans les collèges, comme une simple continuation des opérations arithmétiques élémentaires pour les objets algébriques. Cette conception résultait de l'attention sur les propriétés structurales de ces opérations (par exemple, la commutativité de l'addition et la multiplication) qui sont aussi valables pour les opérations algébriques. En général, dans beaucoup de systèmes d'éducation et en particulier au Mexique, cela a conduit à ce que Chevallard appelle une frontière oubliée $e^{4}$, entre les pensées arithmétique et algébrique. Pendant la décennie des années quatrevingt, la recherche de base en éducation mathématique a prouvé que les deux pensées sont de nature différente, ce qui explique bien des difficultés des élèves pour apprendre le langage algébrique. D'autre part, on considérait la résolution de problèmes comme une simple application de méthodes mathématiques, sans que cela ait eu une place propre dans le curriculum. La part accordée à la géométrie euclidienne fut réduite et on introduisit les thèmes de logique et la théorie des ensembles.

$5 \mathrm{Au}$ Mexique, pendant cette période, le traitement taxonomique et par objectifs des contenus curriculaires a rejoint cette approche structuraliste. Peut-être ce rapprochement explique-t-il les résultats des études réalisées dans notre pays qui montrent une chute de la capacité algorithmique, de maniement algébrique et de résolution de problèmes chez les générations qui ont été formées par cette approche ${ }^{5}$.

6 La réforme de l'éducation de 1993 propose des changements essentiels dans les programmes de mathématiques de l'éducation de base dont, entre autres, une rectification de l'approche structuraliste du curriculum antérieur. Dans la nouvelle approche, on propose un enseignement des mathématiques fondé sur la résolution de problèmes : « apprendre les mathématiques en résolvant des problèmes $»^{6}$. On reprend les connaissances issues de la recherche en mathématiques et de la psychomathématique, en prenant en considération les concepts que l'élève pouvait déjà avoir au sujet des thèmes d'enseignement proposés ainsi que les difficultés rapportées liées à l'apprentissage de ces mêmes thèmes. On insiste sur la différence entre enseignement et processus d'apprentissage grâce à quoi on peut voir le rôle différent des didactiques spécifiques de l'enseignement (par exemple, celle des nombres rationnels ou celle de l'algèbre).

7 A l'école primaire, l'approche par la résolution de problèmes est orientée vers des situations concrètes, pour que les élèves puissent se passer des objets physiques et atteindre un niveau plus abstrait de conceptualisation quand ils comparent leurs points de vue. Ceci va de pair avec l'idée que la connaissance mathématique se construit à partir d'abstractions successives et met en relief son caractère instrumental.

8 Au collège (12-15 ans), on aspire à ce que les élèves élargissent et améliorent leurs capacités à résoudre des problèmes et à les transposer à d'autres domaines ${ }^{7}$. En général, à ce niveau de scolarisation, on cherche le développement des capacités opératoires, de communication et de découverte.

9 On modifie aussi les contenus et la structure du curriculum de l'école primaire; par exemple, on élimine les thèmes de «logique et ensembles", les nombres négatifs sont 
enseignés à partir du collège ; on commence à enseigner les fractions dès les premières années mais c'est au collège qu'elles deviennent complètement opératoires; les propriétés des opérations arithmétiques ne sont démontrées que comme un moyen de faciliter les calculs. Les notions de poids, capacité, surface, temps, longueur et de la distance sont introduites dès la première année d'école primaire. On redécouvre l'utilisation des instruments de tracé géométrique.

$10 \mathrm{Au}$ collège, les contenus sont regroupés en cinq domaines: arithmétique, algèbre, géométrie (avec trigonométrie en troisième année), présentation et traitement de l'information, notions de probabilité. Il est important de mentionner que dans les deux cycles, la géométrie a, de nouveau, une présence importante mais à partir d'une approche qui favorise l'élaboration et la mise à l'épreuve d'hypothèses de la part des élèves. Pour faire la liaison entre école primaire et collège, on introduit des sous-thèmes de préalgèbre qui permettront aux élèves de développer des expériences qui les prépareront à passer à des notions plus abstraites. On incorpore des activités de reconnaissance de modèles et de généralisation et on réduit la manipulation algébrique; on insiste sur le rôle de l'algèbre comme langue de modélisation et de résolution de problèmes.

11 Une partie des stratégies pour introduire la réforme de 1993 dans le système de l'éducation nationale est la refonte des livres gratuits pour l'enseignement primaire ${ }^{8}$ ainsi que la production de livres et de matériels pour le maître, aussi bien au niveau primaire ${ }^{9}$ qu'au collège ${ }^{10}$. Une tendance constante dans ces livres et matériels, dans le cas des mathématiques, est l'insistance sur l'utilisation de «situations de problème» pour introduire des notions nouvelles pour l'élève. Ces situations doivent constituer un défi pour les élèves, en fonction de l'âge auquel elles leur sont présentées et être en rapport avec les autres matières enseignées.

12 Pour mettre en pratique cette approche, le maitre d'école primaire peut utiliser les «Fichiers $»^{11}$ qui contiennent des problèmes et des activités qui suivent cette orientation. De plus, à travers les cours de recyclage du PRONAP ${ }^{12}$, le maître s'initie à la didactique des mathématiques et réalise des activités similaires à celles qu'il proposera aux élèves pour prendre conscience et analyser les difficultés possibles d'apprentissage qu'ils pourraient rencontrer et, en même temps, les amener à disposer de plus d'éléments pour savoir comment les mathématiques aident à interpréter le monde. Un curriculum flexible de mathématiques présente une plus grande difficulté pour le professeur de collège, puisque la programmation des contenus implique une prise de décisions délicate. Le livre Secuencia y organizaciôn de contenidos ${ }^{13}$ oriente le professeur dans cette tâche.

\section{Sciences naturelles}

13 Avant la réforme de 1993, au Mexique comme dans d'autres parties du monde, on était convaincu que si un élève reproduisait une activité scientifique, il acquerrait une certaine connaissance, ce qui favorisait la réalisation d'expériences, pour mettre l'élève en face des phénomènes naturels. On donnait plus d'importance à la "méthode scientifique " comme processus de découverte ${ }^{14}$. D'autre part, on a dit de ce type d'enseignement qu'il voulait former des « petits scientifiques $»^{15}$.

Les recherches en éducation réalisées pour savoir comment les enfants apprennent ont établi les bases d'une nouvelle proposition pour l'enseignement des sciences naturelles ${ }^{16}$. On a constaté qu'il est nécessaire de considérer l'expérience des enfants ainsi que leurs 
conceptions des phénomènes comme point de départ des stratégies d'enseignement ${ }^{17} \mathrm{Il}$ s'agit d'arriver à un rapprochement avec la pensée scientifique de telle sorte que l'élève l'utilise pour élargir sa compréhension du monde, en commençant par les phénomènes présents dans sa vie quotidienne ${ }^{18}$. Il faut questionner ce que l'on pense ${ }^{19}$, ce pour quoi il faut élaborer et réaliser des activités ad hoc. Quant aux expériences, on reconnaît qu'il faut combiner deux stratégies: celle qui permet d'éclairer certains concepts ou lois générales en se basant sur des expériences « traditionnelles » et celle qui essaie d'obtenir que l'élève construise ses connaissances en opposant ses idées aux résultats d'expériences simples ${ }^{20}$. Autrement dit, on vise à ce que les personnes « apprennent bien ${ }^{21}$. En général, dans la vie courante, on n'a pas à appliquer des connaissances scientifiques ou technologiques sophistiquées et il convient donc que l'éducation de base favorise la compréhension de ce qui est essentiel dans l'activité scientifique. Ainsi, le futur citoyen pourra développer les compétences, capacités et attitudes qui lui permettront, au moins, d'avoir des connaissances de base sur son corps et sur le monde où il vit. On considère que cette approche coïncide avec les lignes de conduite mentionnées par l'UNESCO ${ }^{22}$ quant au besoin de former des citoyens « alphabétisés scientifiquement " ${ }^{23}$.

On favorise certains aspects de l'activité scientifique comme :

- apprendre à observer au lieu de simplement « regarder» ;

- réunir des informations de manière systématique ;

- hasarder des réponses et les comparer;

- élaborer et réaliser des expériences ;

- garder un esprit ouvert et critique face à n'importe quelle évidence ;

- développer un langage scientifique.

L'enseignement des sciences naturelles à l'école primaire se base sur quatre principes d'orientation et cinq axes thématiques qui regroupent les contenus.

\section{Principes d'orientation :}

- associer l'acquisition de connaissances sur le monde actuel avec la formation et la pratique d'attitudes et de capacités scientifiques ;

- relier la connaissance scientifique et ses applications techniques ;

- accorder une attention spéciale aux thèmes liés à la protection de l'environnement et de la santé ;

- favoriser la relation de l'apprentissage des sciences naturelles avec le contenu des autres matières.

\section{Axes thématiques :}

- les êtres vivants ;

- l'environnement et sa protection ;

- sciences, technologie et société ;

- le corps humain et la santé ;

- matière, énergie et changement.

Tout ceci est présenté dans les livres à partir des réponses à trois questions de base : pourquoi ? comment? et qu'arriverait-il si... ? Les livres développent les thèmes du niveau auquel ils correspondent en insistant sur leur relation avec la protection de la santé et de l'environnement. Chaque livre est composé de quatre grands ensembles de huit leçons et d'un cinquième qui reprend tout ce qui a été vu pendant l'année, grâce à la réalisation de projets de recherche simples ${ }^{24}$. Chaque ensemble combine axes thématiques et principes d'orientation, montrant par là l'intégration des sciences à partir des 
phénomènes de l'environnement dans la vie quotidienne ou en relation avec le corps humain et la santé. Il existe des leçons spécifiques pour décrire les différents systèmes et organes du corps humain ; au moyen d'activités, on introduit des notions de physique ou d'autres qui sont en relation avec la technologie, la société et l'environnement; de même en troisième année, par exemple, quand on introduit le thème du mouvement, on mentionne les risques de santé qui y sont associés (comme les accidents par coups ou chute), ainsi que les mesures de prévention accessibles à un enfant de neuf ans.

À partir de 1997, on distribuera les livres du maitre qui accompagnent les nouveaux livres des élèves. Ils offriront des suggestions didactiques pour appliquer l'approche proposée dans les programmes en vigueur, en insistant sur l'utilisation de différentes ressources d'information et de matériels bon marché pour comprendre les phénomènes et les concepts qui correspondent à chaque année d'études.

21 L'enseignement au collège est divisé en matières (chimie, biologie, physique et géographie), tout en continuant le développement des compétences, capacités et attitudes, pour approfondir la formulation d'explications scientifiques pour ces domaines de connaissance, en cherchant de façon permanente une interaction entre elles et les phénomènes sociaux. On poursuit encore le but de promouvoir une culture de prévention qui favorise la santé du corps et la protection à l'environnement.

La diffusion de la réforme à ce niveau a été menée à bien, principalement à travers les actions suivantes:

- distribution des livres de chaque matière pour le maître. Ceux-ci offrent des suggestions didactiques pour appliquer l'approche proposée dans les programmes en vigueur, en insistant sur l'utilisation des différentes ressources d'information et de matériels bon marché aidant à comprendre les phénomènes et les concepts qui correspondent à chaque matière ${ }^{25}$;

- ateliers pour les maitres, par matière ;

- distribution de vidéothèques et retransmission de téléconférences sur les utilisations possibles des médias (spécialement les documents vidéo) dans l'enseignement secondaire ;

- cours du programme de recyclage de chimie, biologie, physique et géographie ${ }^{26}$.

La réforme de l'éducation au Mexique qui a commencé en 1992 est un processus qui allie plusieurs éléments étroitement solidaires. La refonte et la distribution des livres gratuits à tous les élèves de l'école primaire jouent un rôle central, comme dans les réformes antérieures. Cependant, l'incorporation comme partie intégrante de l'éducation de base du cycle de la maternelle ainsi que du collège ont obligé les stratégies, les appuis et les ressources d'augmenter. La réorganisation structurale du système, les réformes légales, la reformulation des contenus, la rénovation des matériels (pour les élèves, les maîtres et les parents d'élèves), le programme de perfectionnement des maîtres, la réforme des écoles normales sont des éléments qui permettront d'introduire les innovations qui sont en totalité le fruit de la réforme, en prêtant attention à tous les secteurs qui composent le système de l'Éducation nationale - c'est-à-dire ses dimensions académiques, administratives et organisationnelles.

Dans ce contexte, les innovations touchant à l'enseignement des sciences naturelles et des mathématiques se rapprochent des tendances générales des réformes de l'éducation au niveau mondial. C'est-à-dire que le but recherché est de promouvoir une éducation toujours plus pratique, plus proche de la réalité des élèves, qui les aide à interpréter le monde et qui leur permette d'établir des relations entre les différents domaines du savoir. 
Cependant, il est encore important de développer des programmes de suivi pour savoir comment ces innovations sont appliquées dans la salle de classe par les maitres et comment elles sont comprises par les élèves.

\section{NOTES}

3. Black, P. Atkin, J.M. Atkin, Changing the Subject - Innovations in Science Mathematics and Technology Education, London/New York, OECD-Routledge, 1996.

4. Y. Chevallard, "Le passage de l'arithmétique à l'algébrique dans l'enseignement des mathématiques ", Petit x (France), n 5, 1983, p. 51-94.

5. M. Trigueros, S. Ursini, A. Reyes, «College Student's Conceptions of variable », Proceedings of the XXth Conference of the International Group for the Psychology of Mathematics Education, Valencia, Espana, vol. 4, p. 315-322.

6. SEP, Guia de estudio. La enseñanza de las Matematicas en la escuela secundaria, México, 1995.

7. SEP, Plan y programas. Education Bâsica Secundaria, México, 1993.

8. SEP, Maternãticas, $1^{\circ}, 3^{\circ}, 5^{\circ}$ grados, Mexico, 1993. SEP, Maternâticas, $2^{\circ}, 4^{\circ}, 6^{\circ}$ grados, México, 1994.

9. SEP, Libro para el maestro de Matemâticas, $1^{\circ}, 2^{\circ}, 3^{\circ}, 4^{\circ}, 5^{\circ}, 6^{\circ}$ grados ; México, 1994 ; SEP, Avance programàtico, Matemâticas $1^{\circ}, 2^{\circ}, 3^{\circ}, 4^{\circ}, 5^{\circ}, 6^{\circ}$ grados, México, 1994.

10. SEP, Libro para el maestro de matemâticas, Mexico, 1994.

11. SEP, Fichera Matemãticas, $1^{\circ}, 3^{\circ}, 5^{\circ}$, grados, México, 1994 ; SEP, Fichera Matemãticas, $2^{\circ}, 4^{\circ}, 6^{\circ}$ grados , México, 1995.

12. SEP, Lecturas. La ensenanza de las Matemâticas en la escuela sucundaria, México, 1995.

13. SEP, Secuencia y organizaciôn de contenidos, México, 1994.

14. M.A Candela, «Los libros de texto gratuitos de ciencias naturales y la investigaciôn en ensenanza de las ciencias ", Avance y Perspectiva, vol. 8, $\mathrm{n}^{\circ}$ 37, Mexico, 1989.

15. Ibid. note 1.

16. R. Driver et al, Ideas cientificas en la infancia y en la adolescencia, Morata, Madrid, 1989.

17. D.P. Ausbel et al, Psicologia Educativa. Un punto de vista cognoscitivo, México, Ed. Trillas, 1993.

18. SEP, Libro para el maestro de Quimica, México, 1994 ; SEP, Libro para el maestro de Biologia, México, 1994 ; SEP, Libro para el maestro de Fisica, México, 1996.

19. A. Giordan, G. Vecchi, Les origines du savoir, Neuchâtel, Delachaux et Niestlé, 1994.

20. A. di Sessa, Toward an Epistemology of Physics. Cognition and Instruction, vol. 10 (2-3), p. 105-205, États-Unis, 1993.

21. G. Claxton, Educar Mentes curiosas, Madrid, Visor, 1994.

22. UNESCO, Declaracion del proyecto 2000+, Foro International sobre el Conocimiento Cientifico y Technolôgico para todos, Paris, 1993.

23. J. Rutheford, A. Ahlgren, Science for All Americans, New York, Oxford University Press, 1990.

24. SEP, Ciencias Naturales.Tercer grado, México, 1996 ; SEP, Ciencias Naturales, $4^{\circ}, 5^{\circ}$ grados, México, 1997 (en préparation).

25. SEP, Libro para el maestro de Quimica, México, 1994; SEP, Libro para el maestro de Biologia, México, 1994 ; SEP, Libro para el maestro de Fisica, México, 1996.

26. SEP, Guia de estiudio. La ensenanza de la Quimica en la escuela secundaria, México 1995 ; SEP, Guia de estudio. La ensenanza de la Biologia en la escuela secundaria, México 1995 ; SEP, Lecturas. La ensenanza de la Quimica en la escuela secundaria. México 1995 ; SEP, Guia de estudio. La ensenanza de la 
Geografia en la escuela secundaria, México, 1995 ; SEP, Lecturas. La ensenanza de la Geografia en la escuela secundaria, México, 1995 ; SEP, Gui'a de estudio. La ensenanza de la Fisica en la escuela secundaria, México, 1997 ; SEP, Lecturas. La ensenanza de la Fisica en la escuela secundaria, México, 1997.

\section{RÉSUMÉS}

Dans le cadre de sa réforme de l'éducation, le Mexique a restructuré les programmes de mathématiques et de sciences naturelles. De nouveaux manuels ont été distribués gratuitement aux élèves et aux professeurs. Se basant sur la résolution de problèmes ou la réalisation d'expériences, cette approche des disciplines scientifiques doit favoriser la compréhension par tous de ce qui est essentiel dans l'activité scientifique.

\section{INDEX}

Mots-clés : enseignant, manuel d'enseignement, programme d'enseignement, qualité de l'éducation, réforme de l'enseignement

Index géographique : Mexique

\section{AUTEURS}

\section{ELISA BONILLA}

Secretaria de Educación Pública (SEP), ministerío de Educación pública, Mexico, Mexique.

\section{ARMANDO SÁNCHEZ}

Secretaria de Educación Pública (SEP) ministerio de Educación pública, Centro de Investigación y Estydios Avanzados (CINVESTAV), Mexico, Mexique.

\section{TERESA ROJANO}

Secretaria de Educación Pública (SEP) ministerio de Educación pública, Centro de Investigación y Estydios Avanzados (CINVESTAV), Mexico, Mexique.

\section{JOSÉ ANTONIO CHAMIZO}

Colegio Madrid et Universidad nacional Autónoma, Mexico, Mexique. 